\title{
Effect of different types of statins on kidney function decline and proteinuria: a network meta- analysis
}

\author{
K. Esmeijer ${ }^{1,2^{*}}$, Olaf M. Dekkers ${ }^{2,3}$, Johan W. de Fijter ${ }^{1}$, Friedo W. Dekker ${ }^{2} \&$ \\ Ellen K. Hoogeveen $\mathbb{1}^{1,2,4}$
}

Previous studies showed that statins reduce the progression of kidney function decline and proteinuria, but whether specific types of statins are more beneficial than others remains unclear. We performed a network meta-analysis of randomized controlled trials (RCT) to investigate which statin most effectively reduces kidney function decline and proteinuria. We searched MEDLINE, Embase, Web of Science, and the Cochrane database until July 13, 2018, and included 43 RCTs (>110,000 patients). We performed a pairwise random-effects meta-analysis and a network meta-analysis according to a frequentist approach. We assessed network inconsistency, publication bias, and estimated for each statin the probability of being the best treatment. Considerable heterogeneity was present among the included studies. In pairwise meta-analyses, 1-year use of statins versus control reduced kidney function decline by $0.61(95 \%-\mathrm{Cl}: 0.27 ; 0.95) \mathrm{mL} / \mathrm{min} / 1.73 \mathrm{~m}^{2}$ and proteinuria with a standardized mean difference of $-0.58(95 \%-\mathrm{Cl}:-0.88 ;-0.29)$. The network meta-analysis for the separate endpoints showed broad confidence intervals due to the small number available RCTs for each individual comparison. In conclusion, 1-year statin use versus control attenuated the progression of kidney function decline and proteinuria. Due to the imprecision of individual comparisons, results were inconclusive as to which statin performs best with regard to renal outcome.

Chronic kidney disease (CKD) is an increasing global health burden owing to population ageing and unhealthier lifestyle ${ }^{1}$. Up to $11 \%$ of the European population aged $45 \mathrm{y}$ or older has CKD stage 3, defined as an estimated glomerular filtration rate (eGFR) below $60 \mathrm{~mL} / \mathrm{min} / 1.73 \mathrm{~m}^{2}$. CKD is an independent risk factor for cardiovascular morbidity and mortality ${ }^{3}$. Nowadays, the most important causes of CKD are cardiovascular disease, hypertension, diabetes, smoking, and hypercholesterolemia ${ }^{4,5}$. Generally, patients with symptomatic cardiovascular disease are prescribed cholesterol-lowering medication for secondary cardiovascular prevention. The latest KDIGO guideline on lipid management in CKD, recommends treatment with a statin in all non-dialysis dependent CKD patients $\geq 50$ years with an eGFR below $60 \mathrm{~mL} / \mathrm{min} / 1.73 \mathrm{~m}^{2}$ or with at least $30 \mathrm{mg} / \mathrm{g}$ albuminuria, independent of serum cholesterol levels, which is also stated by the $2016 \mathrm{ESC} / \mathrm{EAS}$ guidelines ${ }^{6,7}$. Younger patients should use a statin in case of elevated cardiovascular risk, such as diabetes or coronary heart disease. Finally, statins should be continued, but not initiated, in patients on dialysis ${ }^{6}$. Multiple meta-analyses studied the effect of statins on renal outcomes. Recently, a meta-analysis by Su et al. concluded that statin users $v s$ nonusers have a slower rate of kidney function decline and less proteinuria ${ }^{8}$.

Targeted prevention of kidney function decline is important to improve life expectancy and quality of life. However, it remains unclear whether specific types of statins are more beneficial than others regarding slowing down kidney function decline and lowering proteinuria. Various statins have different characteristics in terms of half-life, structure, lipophilicity, and potency ${ }^{9}$. We therefore performed a network meta-analysis of randomized controlled trials in adults that compare any statin with another statin or control treatment, to investigate which statin most effectively reduces kidney function decline or proteinuria. Network meta-analyses take into account

${ }^{1}$ Department of Nephrology, Leiden University Medical Center, Leiden, The Netherlands. ${ }^{2}$ Department of Clinical Epidemiology, Leiden University Medical Center, Leiden, The Netherlands. ${ }^{3}$ Department of Endocrinology, Leiden University Medical Center, Leiden, The Netherlands. ${ }^{4}$ Department of Nephrology, Jeroen Bosch Hospital, Den Bosch, The Netherlands. *email: k.esmeijer@lumc.nl 
both direct and indirect evidence of multiple comparisons in a treatment network, and provide information on which treatment performs best. These results may inform future guidelines about prevention of CKD and slowing down its progression.

\section{Methods}

Systematic literature review. We performed a systematic review of the literature, searching MEDLINE, Embase, Web of Science, and the Cochrane Library, on July $13^{\text {th }}, 2018$. Eligible studies were randomized controlled trials (RCT) in adults (patients $\geq 18$ years) with a follow-up duration of at least one year, that included at least 10 patients per trial arm, and reported on changes in eGFR and/or proteinuria. The intervention of interest was statin therapy, the comparator either another statin, no intervention, cholesterol lowering diet, or placebo. In the entire manuscript, control treatment refers to any non-statin intervention. Combination therapy of statin with ezetimibe was also considered. A detailed outline of the search strategy is provided in the Supplemental Data, Appendix. Titles and abstracts were screened and relevant articles were read in full by two reviewers (KE and EH). Conference abstracts were excluded. No language restrictions were imposed. Post-hoc analyses of RCTs were only included when outcomes according to the original randomization group could be derived. In case of duplicate publications, we selected the publication that reported the data of interest most completely. References of included studies were additionally screened for relevant RCTs. We reported the results according to the Preferred Reporting Items for Systematic Reviews and Meta-analyses (PRISMA) guidelines for network meta-analyses ${ }^{10}$. The protocol for this meta-analysis was registered at PROSPERO: registration number CRD42018099613 ${ }^{11}$.

Outcome measures. The outcomes of interest were annual change of estimated glomerular filtration rate (eGFR) and proteinuria. Kidney function estimates calculated by the Cockroft-Gault formula, the Modification of Diet in Renal Disease (MDRD) formula, or Chronic Kidney Disease Epidemiology Collaboration (CKD-EPI) equation were pooled. If change of kidney function or proteinuria was not reported, it was calculated by subtracting the baseline value from follow-up. The standard deviation (SD) of change was calculated using the SDs of eGFR or proteinuria at baseline and follow-up, according to the following formula ${ }^{12}$ :

$$
\mathrm{SD}_{\text {change }}=\sqrt{\mathrm{SD}_{0}^{2}+\mathrm{SD}_{1}^{2}-\left(2 * \operatorname{Corr}_{*} \mathrm{SD}_{0} \mathrm{SD}_{1}\right)}
$$

where $\mathrm{SD}_{0}$ and $\mathrm{SD}_{1}$ represent the $\mathrm{SD}$ of baseline and follow-up, respectively, and Corr represents a correlation coefficient, which describes the similarity between baseline and follow-up measurements. The correlation coefficient was derived from studies that reported both baseline and follow-up eGFR or proteinuria with an SD, and change in eGFR or proteinuria with SD, according to the following formula ${ }^{12}$ :

$$
\text { Corr }=\frac{\mathrm{SD}_{0}^{2}+\mathrm{SD}_{1}^{2}+\mathrm{SD}_{\text {change }}{ }^{2}}{2 * \mathrm{SD}_{0 *} \mathrm{SD}_{1}}
$$

Based on data from three intervention studies investigating the effect of statins on kidney function, and data from the Alpha Omega Trial, we assumed a correlation coefficient between baseline and follow-up eGFR of $0.8^{13-}$ ${ }^{16}$. In the main analysis we compared change of eGFR or proteinuria after 12 months for statin users $v s$ control treatment. If no data were reported on change in eGFR or proteinuria after one year, we used the available data to calculate an annual change assuming a linear decline in line with the results of a recent study ${ }^{17}$.

Data extraction and quality assessment. Data extraction was performed by two independent reviewers (KE and $\mathrm{EH}$ ) who used a standard form. Discrepancies were resolved by discussion or by consulting a third reviewer $(\mathrm{OD})$. We extracted the following data: study name, study year, trial acronym, duration, population type, treatment arms, sample size, mean age, sex (\% males), diabetes (\%), hypertension (\%), mean systolic and diastolic blood pressure, use of renin-angiotensin system (RAS) blocking drugs (\%), low-density lipoprotein (LDL) level at baseline and follow-up, baseline and follow-up eGFR, change in eGFR, baseline and follow-up proteinuria, and change in proteinuria. When the outcome of interest was not reported in a table or text, we extracted the exact numbers from figures.

The Cochrane Collaboration Risk of Bias tool was used to assess potential sources of bias: selection, performance, detection, attrition and reporting bias ${ }^{18}$. We scored per included RCT each type of bias as follows: low, high, or unclear risk of bias. Risk of bias was scored high in case of broken randomization, absent blinding of participants, absence of allocation concealment, and in case of large number of missing outcome data, or exclusion of patients. Since the outcome of interest was based on laboratory measurements, we considered for all RCTs, including the open-label RCTs, the risk of bias "low" with regard to blinding of outcome assessment.

Statistical analysis. First, we performed a pairwise random-effects meta-analysis for the effect of statin $v s$ control on eGFR and proteinuria decline. For eGFR decline we used the weighted mean difference (WMD) as measure for the pooled estimates. For proteinuria we estimated standardized mean differences (SMD) to account for different methods to express proteinuria: urinary albumin to creatinine ratio, urinary protein excretion, urinary albumin excretion, or log-transformed protein excretion. Statistical heterogeneity was assessed by the $\mathrm{I}^{2}$-statistic, which quantifies the variation across studies due to heterogeneity rather than chance ${ }^{19}$. We used meta-regression to evaluate whether heterogeneity could be explained by age, sex, diabetes, blood pressure, baseline LDL, change in LDL, or risk of bias. Finally, we assessed the presence of publication bias visually with a funnel plot and formally by the Egger's test ${ }^{20,21}$. This rank-based method estimates the number and outcomes of missing unpublished studies, and adjusts the estimate after incorporating these theoretical studies. 


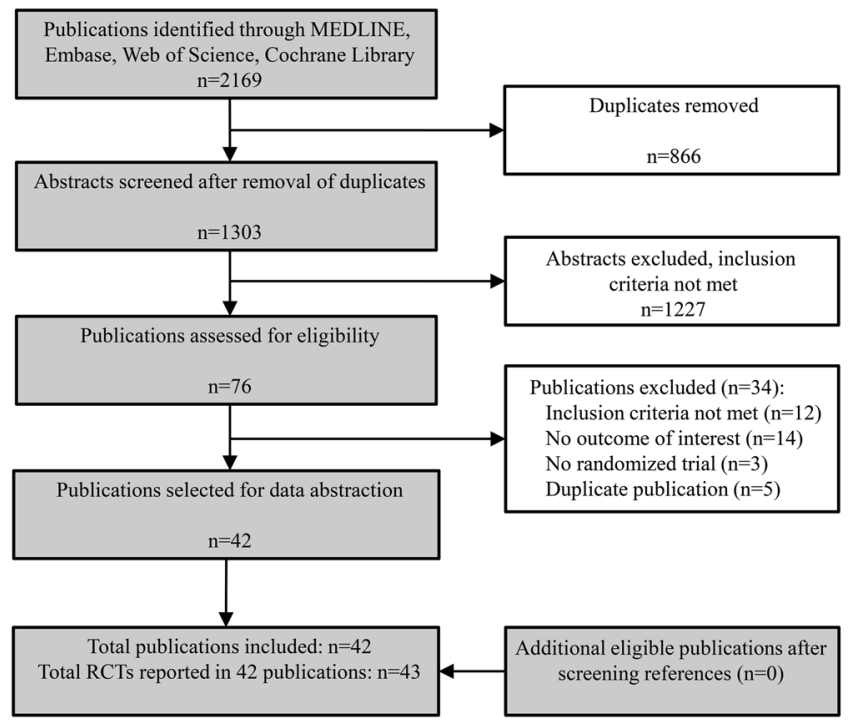

Figure 1. Flow chart of literature search and included full text publications. All included publications were included in quantitative analyses, depending on the reported endpoint(s).

Second, we performed a random-effects network meta-analysis, following a frequentist approach. In case multiple dosages were reported, we analyzed high and low statin dosages as separate treatments. We took as outcome the WMD of annual kidney function decline and change of proteinuria expressed as SMD. We checked for transitivity and consistency. Transitivity was judged clinically; consistency was judged formally ${ }^{22}$. We tested for possible inconsistency globally using a $\chi^{2}$-test, and locally by calculating inconsistency factors for each comparison in closed loops. In case of minor inconsistencies, possible reasons for inconsistency were considered. Furthermore, we estimated for each statin, compared to control, the treatment effect with $95 \%$-confidence intervals and prediction intervals. The prediction interval represents the expected range of true effects in similar (future) studies, and will be broader than the confidence interval in case of high heterogeneity ${ }^{23}$. Finally, for each statin, with or without ezetimibe, we calculated the surface under the cumulative ranking (SUCRA) line. We used the SUCRA to provide a hierarchic overview of treatments, and to give an impression of the most efficacious treatments ${ }^{24}$. The SUCRA takes into account for every treatment the cumulative probabilities of all possible rankings. If a treatment always ranks first, the SUCRA is $100 \%$ (or 1), and $0 \%$ (or 0 ) if it always ranks last $\mathrm{t}^{25}$.

We repeated the analyses excluding RCTs with a total sample size $<100$ patients or stratified by open-label (yes/no) or post-hoc (yes/no) status. Subgroup analyses were not considered if too few RCTs remained to form a network. All statistical analyses were performed using STATA Statistical Software version 14 (Statacorp, Texas, USA), and the StataNMA package ${ }^{26}$.

\section{Results}

Characteristics of included studies. After removing duplicate RCTs, 1303 titles and abstracts were screened for eligibility; 76 full publications were assessed. Finally, 43 RCTs comprising over 110,000 patients reported in 42 publications were included (Fig. 1). Of these 42 publications, 40 were in English, one was Russian ${ }^{27}$, and one Japanese ${ }^{28}$. In total, 40 RCTs reported about the effect of statins on change of eGFR ${ }^{13-15,27,29-63}$, of which 30 compared a statin to control, and 10 compared two or more statins with each other. The effect of statins on proteinuria was reported in $25 \mathrm{RCTs}^{13,14,28,29,32-34,36,39,45,46,48-54,57,60,62-65}$, of which 19 compared a statin to control intervention, and six compared two or more statins. Characteristics of included RCTs are shown in Table 1. The included RCTs investigated seven different statins with varying dosages, and in three RCTs a statin was combined with ezetimibe ${ }^{40,46,48}$. Of all included RCTs, 11 comprised coronary heart disease patients, 11 comprised CKD patients, and 11 comprised diabetes mellitus type 2 patients. The mean age of the enrolled patients in most RCTs was over 50 years and about $66 \%$ were men. The unweighted mean (range) of baseline LDL-cholesterol from all individual RCTs was $3.7(2.2-7.8) \mathrm{mmol} / \mathrm{L}$, and statin compared to control treatment led to a mean (SD) $27 \%$ (9\%) reduction of the serum LDL level. The majority of RCTs had a low risk of bias (Supplementary Fig. S1). However, about a $44 \%$ of all RCTs was open-label and about $25 \%$ were post-hoc analyses.

Pairwise comparison: statins and eGFR decline. Except for two medium sized trials (Yasuda et al., and Nanayakkara et al.), effect estimates of all RCTs showed a protective effect of statin on eGFR decline e $^{53,62}$. Random-effects meta-analysis showed that statin use, compared to control, led to a $0.61(95 \% \mathrm{CI} 0.27 ; 0.95) \mathrm{mL} /$ $\mathrm{min} / 1.73 \mathrm{~m}^{2}$ slower annual eGFR decline (Fig. 2). When only RCTs with a sample size of at least 100 patients $(n=16)$ were analyzed, the beneficial effect of statin treatment on annual eGFR decline was 0.58 (95\%-CI 0.23; $0.92) \mathrm{mL} / \mathrm{min} / 1.73 \mathrm{~m}^{2}$. Heterogeneity between RCTs was high, with an $\mathrm{I}^{2}$ of $96 \%$. Meta-regression showed that higher systolic blood pressure at baseline was significantly associated with smaller effects of statins, explaining $40 \%$ of the between-study variance. We found no evidence for interaction between diabetes and statins with regard to the beneficial effect on kidney function decline. Age, sex, serum LDL level, or change in LDL, had no 


\begin{tabular}{|c|c|c|c|c|c|c|c|c|c|c|c|c|}
\hline \multirow[b]{2}{*}{$\begin{array}{l}\text { Author, year } \\
\text { Study name }\end{array}$} & \multirow[b]{2}{*}{ Population } & \multirow[b]{2}{*}{ Intervention } & \multicolumn{8}{|c|}{ Mean baseline characteristics per RCT } & \multicolumn{2}{|c|}{$\begin{array}{l}\text { Outcome [annual change } \\
\text { (SD)] } \\
\text { per intervention }\end{array}$} \\
\hline & & & \begin{tabular}{|l|} 
Sample \\
size \\
$(\mathbf{n})$
\end{tabular} & $\begin{array}{l}\text { Follow- } \\
\text { up } \\
(y)\end{array}$ & $\begin{array}{l}\text { Age } \\
\text { (y) }\end{array}$ & \begin{tabular}{|l|} 
Male \\
sex \\
$(\%)$ \\
\end{tabular} & $\begin{array}{l}\text { Diabetes } \\
\text { (\%) }\end{array}$ & \begin{tabular}{|l|} 
Blood \\
pressure \\
$(\mathrm{mmHg})$
\end{tabular} & \begin{tabular}{|l|} 
eGFR \\
$(\mathrm{mL} /$ \\
$\left.\mathrm{min} / 1.73 \mathrm{~m}^{2}\right)$
\end{tabular} & $\begin{array}{l}\text { LDL } \\
(\mathrm{mmol} / \mathrm{L})\end{array}$ & eGFR & Proteinuria \\
\hline \multicolumn{13}{|l|}{ (measure) } \\
\hline Abe, 2015 & CKD & $\begin{array}{l}\text { Rosuvastatin } \\
2.5 \mathrm{mg} \\
\text { Pitavastatin } \\
\text { mean } 1.4 \mathrm{mg} \\
\end{array}$ & 134 & 1 & 70 & 58 & 44 & . & 58 & 3.6 & $\begin{array}{l}2.80(12.1) \\
0.90(13.8)^{\mathrm{a}}\end{array}$ & $\begin{array}{l}-392(802) \\
-250(707)^{\mathrm{a}} \\
(\mathrm{UACR})\end{array}$ \\
\hline $\begin{array}{l}\text { Amarenco, } 2014 \\
\text { SPARCL }\end{array}$ & Stroke, TIA & \begin{tabular}{|l|} 
Atorvastatin \\
$80 \mathrm{mg}$ \\
Placebo \\
\end{tabular} & 4719 & 5 & 63 & 60 & 17 & $139 / 82$ & 66 & 3.4 & $\begin{array}{l}0.96(13.1) \\
-0.50(13.1)^{\mathrm{b}}\end{array}$ & $\cdot$ \\
\hline $\begin{array}{l}\text { Athyros, } 2004 \\
\text { GREACE }\end{array}$ & CHD & $\begin{array}{l}\text { Atorvastatin } \\
\text { mean } 24 \mathrm{mg} \\
\text { Control }\end{array}$ & 1600 & 4 & 58 & 78 & 20 & $123 / 75$ & 77 & . & $\begin{array}{l}2.00(2.0) \\
-0.75(1.8)^{\mathrm{a}}\end{array}$ & \\
\hline $\begin{array}{l}\text { Atthobari, } 2006 \\
\text { PREVEND-IT }\end{array}$ & $\begin{array}{l}\text { General } \\
\text { population }\end{array}$ & $\begin{array}{l}\text { Pravastatin } \\
40 \mathrm{mg} \\
\text { Placebo }\end{array}$ & 788 & 4 & 52 & 66 & 3 & $131 / 76$ & 76 & 4.1 & $\begin{array}{l}0.15(3.7) \\
-0.25(1.9)^{\mathrm{a}}\end{array}$ & $\begin{array}{l}-0.02(0.07) \\
-0.03(0.08)^{\mathrm{ac}} \\
(\mathrm{UAE})\end{array}$ \\
\hline Bianchi, 2003 & CKD & $\begin{array}{l}\text { Atorvastatin } \\
40 \mathrm{mg} \\
\text { Placebo }\end{array}$ & 56 & 1 & 56 & 47 & 0 & $133 / 85$ & 50 & 5.1 & $\begin{array}{l}-1.00(5.9) \\
-5.80(6.0)^{\mathrm{a}}\end{array}$ & $\begin{array}{l}-1.0(0.47) \\
0.3(0.47)^{\mathrm{a}} \\
(\mathrm{UPE})\end{array}$ \\
\hline Castelao, 1993 & Transplant & $\begin{array}{l}\text { Lovastatin } \\
20 \mathrm{mg} \\
\text { Simvastatin } \\
10 \mathrm{mg} \\
\end{array}$ & 51 & 1 & 44 & 69 & . & . & 52 & 4.9 & $\begin{array}{l}-1.00(16.6) \\
-4.60(15.3)\end{array}$ & $\begin{array}{l}0.38(1.9) \\
0.31(1.1) \\
\text { (UPE) }\end{array}$ \\
\hline $\begin{array}{l}\text { Colhoun, } 2009 \\
\text { CARDS }\end{array}$ & DM2 & $\begin{array}{l}\text { Atorvastatin } \\
20 \mathrm{mg} \\
\text { Placebo }\end{array}$ & 2838 & 4 & 62 & 68 & 100 & $144 / 83$ & 64 & 3.0 & $\begin{array}{l}0.48(2.7) \\
0.30(2.6)\end{array}$ & $\cdot$ \\
\hline Dalla Nora, 2003 & DM2 & $\begin{array}{l}\text { Atorvastatin } \\
10 \mathrm{mg} \\
\text { Placebo }\end{array}$ & 25 & 1 & 65 & 60 & 100 & . & . & 3.5 & . & \begin{tabular}{|l|}
$2.0(1.9)$ \\
$6.0(1.9)^{\mathrm{d}}$ \\
$(\mathrm{AER})$ \\
\end{tabular} \\
\hline $\begin{array}{l}\text { Deedwania, } 2015 \\
\text { SAGE }\end{array}$ & CAD & $\begin{array}{l}\text { Atorvastatin } \\
80 \mathrm{mg} \\
\text { Pravastatin } \\
40 \mathrm{mg} \\
\end{array}$ & 868 & 1 & 72 & 69 & 23 & . & 62 & 3.8 & $\begin{array}{l}2.38(10.4) \\
0.18(10.3)^{b}\end{array}$ & . \\
\hline Fassett, 2010 & ADPKD & $\begin{array}{l}\text { Pravastatin } \\
20 \mathrm{mg} \\
\text { Control } \\
\end{array}$ & 60 & 2 & 51 & 39 & . & $133 / 86$ & 55 & 3.3 & $\begin{array}{l}-0.31(10.4) \\
-1.34(12.2)\end{array}$ & $\begin{array}{l}-0.04(0.20) \\
0.01(0.09) \\
\text { (UPE) } \\
\end{array}$ \\
\hline $\begin{array}{l}\text { Fassett, } 2010 \\
\text { LORD }\end{array}$ & CKD & $\begin{array}{l}\text { Atorvastatin } \\
10 \mathrm{mg} \\
\text { Placebo }\end{array}$ & 132 & 3 & 60 & 65 & 8 & $143 / 81$ & 31 & 3.4 & $\begin{array}{l}-1.04(3.84) \\
-1.47(3.74)\end{array}$ & $\begin{array}{l}-0.39(0.71) \\
-0.14(0.85) \\
\text { (UPE) } \\
\end{array}$ \\
\hline $\begin{array}{l}\text { Fellstrom, } 2004 \\
\text { ALERT }\end{array}$ & Transplant & $\begin{array}{l}\text { Fluvastatin } \\
40 \mathrm{mg} \\
\text { Placebo }\end{array}$ & 439 & 5 & 50 & 66 & 19 & $144 / 86$ & 52 & 4.1 & $\begin{array}{l}-0.93(8.9) \\
-1.87(8.3)^{\mathrm{a}}\end{array}$ & \\
\hline Fried, 2001 & DM1 & $\begin{array}{l}\text { Simvastatin } \\
10 \mathrm{mg} \\
\text { Placebo }\end{array}$ & 39 & 1.5 & 32 & 56 & 100 & . & . & 3.3 & . & $\begin{array}{l}0.09(0.44) \\
0.14(0.66)^{\mathrm{d}} \\
(\text { AER) }\end{array}$ \\
\hline Gheith, 2002 & $\begin{array}{l}\text { Nephrotic } \\
\text { syndrome }\end{array}$ & $\begin{array}{l}\text { Fluvastatin } \\
20 \mathrm{mg} \\
\text { Control } \\
\end{array}$ & 43 & 1 & 23 & 42 & . & . & 107 & 7.8 & $\begin{array}{l}-4.80(28.8) \\
-35.4(29.4)^{\mathrm{a}}\end{array}$ & $\begin{array}{l}-6.0(2.3) \\
-2.0(2.4)^{\mathrm{de}} \\
(\mathrm{UPE}) \\
\end{array}$ \\
\hline $\begin{array}{l}\text { Haynes, } 2014 \\
\text { SHARP }\end{array}$ & CKD & $\begin{array}{l}\text { Simvastatin } 20 \\
\text { mg/eze } \\
\text { Placebo }\end{array}$ & 5037 & 4 & 63 & 62 & 23 & $139 / 80$ & 27 & 2.9 & $\begin{array}{l}-1.66(3.5) \\
-1.83(3.5)\end{array}$ & . \\
\hline $\begin{array}{l}\text { Holme, } 2010 \\
\text { IDEAL }\end{array}$ & MI & $\begin{array}{l}\text { Atorvastatin } \\
80 \mathrm{mg} \\
\text { Simvastatin } \\
20 \mathrm{mg} \\
\end{array}$ & 8888 & 4.8 & 62 & 81 & 12 & 137.80 & 68 & 3.1 & $\begin{array}{l}0.01(2.7) \\
0.34(2.7)\end{array}$ & . \\
\hline $\begin{array}{l}\text { HPS, } 2003 \\
\text { HPS }\end{array}$ & DM & $\begin{array}{l}\text { Simvastatin } \\
40 \mathrm{mg} \\
\text { Placebo }\end{array}$ & 20536 & 4.8 & 64 & 76 & 29 & $144 / 81$ & . & 3.3 & $\begin{array}{l}-1.23(1.86) \\
-1.40(1.83)\end{array}$ & \\
\hline $\begin{array}{l}\text { Huskey, } 2009 \\
4 \mathrm{~S}\end{array}$ & CHD & $\begin{array}{l}\text { Simvastatin } \\
20 \mathrm{mg} \\
\text { Placebo } \\
\end{array}$ & 3842 & 5.5 & 58 & 80 & 4 & $139 / 83$ & 76 & 4.9 & $\begin{array}{l}-0.34(7.4) \\
-0.41(7.4)^{\mathrm{f}}\end{array}$ & . \\
\hline $\begin{array}{l}\text { Kendrick, } 2010 \\
\text { AFCAPS/Tex }\end{array}$ & $\begin{array}{l}\text { Primary } \\
\text { prevention }\end{array}$ & $\begin{array}{l}\text { Lovastatin } \\
20 \mathrm{mg} \\
\text { Placebo }\end{array}$ & 4994 & 5.3 & 58 & 85 & 2 & $138 / 78$ & 87 & 3.8 & $\begin{array}{l}-1.30(3.5) \\
-1.40(3.5)\end{array}$ & \\
\hline $\begin{array}{l}\text { Kimura, } 2017 \\
\text { ASUCA }\end{array}$ & CKD & $\begin{array}{l}\text { Atorvastatin } \\
5-20 \mathrm{mg} \\
\text { Control }\end{array}$ & 334 & 2 & 63 & 64 & 34 & $133 / 77$ & 55 & 3.7 & $\begin{array}{l}-1.15(4.4) \\
-1.30(4.4)\end{array}$ & $\begin{array}{l}0.3(1.3) \\
-0.2(1.3) \\
\log (\text { UAE })\end{array}$ \\
\hline Kimura, 2012 & DM2 & $\begin{array}{l}\text { Pitavastatin } \\
2 \mathrm{mg} \\
\text { Pravastatin } \\
10 \mathrm{mg}\end{array}$ & 83 & 1 & 65 & 57 & 100 & $132 / 76$ & 74 & 3.4 & $\begin{array}{l}-2.0(9.0) \\
-0.5(9.5)^{\mathrm{b}}\end{array}$ & $\begin{array}{l}-50(150) \\
25(175)^{b} \\
\text { (UACR) }\end{array}$ \\
\hline
\end{tabular}




\begin{tabular}{|c|c|c|c|c|c|c|c|c|c|c|c|c|}
\hline \multirow[b]{2}{*}{$\begin{array}{l}\text { Author, year } \\
\text { Study name }\end{array}$} & \multirow[b]{2}{*}{ Population } & \multirow[b]{2}{*}{ Intervention } & \multicolumn{8}{|c|}{ Mean baseline characteristics per RCT } & \multicolumn{2}{|c|}{$\begin{array}{l}\text { Outcome [annual change } \\
\text { (SD)] } \\
\text { per intervention }\end{array}$} \\
\hline & & & \begin{tabular}{|l|}
$\begin{array}{l}\text { Sample } \\
\text { size } \\
(\mathbf{n})\end{array}$ \\
\end{tabular} & $\begin{array}{l}\text { Follow- } \\
\text { up } \\
(\mathrm{y})\end{array}$ & $\begin{array}{l}\text { Age } \\
(y)\end{array}$ & \begin{tabular}{|l|} 
Male \\
sex \\
$(\%)$ \\
\end{tabular} & \begin{tabular}{|l} 
Diabetes \\
$(\%)$
\end{tabular} & \begin{tabular}{|l|}
$\begin{array}{l}\text { Blood } \\
\text { pressure } \\
(\mathrm{mmHg})\end{array}$ \\
\end{tabular} & \begin{tabular}{|l|} 
eGFR \\
$(\mathrm{mL} /$ \\
$\left.\mathrm{min} / 1.73 \mathrm{~m}^{2}\right)$ \\
\end{tabular} & $\begin{array}{l}\text { LDL } \\
(\mathrm{mmol} / \mathrm{L})\end{array}$ & eGFR & Proteinuria \\
\hline Kinouchi, 2013 & Dyslipidemia & \begin{tabular}{|l|} 
Fluvastatin \\
20 mg \\
Fluvastatin 20 \\
mg/eze \\
\end{tabular} & 54 & 1 & 54 & 67 & 6 & $140 / 90$ & 71 & 4.1 & \begin{tabular}{|l}
$-4.10(7.7)$ \\
$4.10(6.4)$
\end{tabular} & $\begin{array}{l}22.5(72.4) \\
-44.7(74.5)^{\mathrm{d}} \\
(\mathrm{UAE})\end{array}$ \\
\hline $\begin{array}{l}\text { Koren, } 2009 \\
\text { ALLIANCE }\end{array}$ & CHD & $\begin{array}{l}\text { Atorvastatin } \\
\text { mean } 41 \mathrm{mg} \\
\text { Control }\end{array}$ & 2442 & 4.5 & 61 & 82 & 22 & $134 / 79$ & 73 & 3.8 & $\begin{array}{l}0.18(6.4) \\
-0.30(7.2)\end{array}$ & . \\
\hline Kouvelos, 2015 & $\begin{array}{l}\text { Vascular } \\
\text { surgery }\end{array}$ & \begin{tabular}{|l|} 
Rosuvastatin \\
$10 \mathrm{mg}$ \\
Rosuvastatin \\
$10 \mathrm{mg} /$ eze \\
\end{tabular} & 262 & 1 & 71 & 90 & 30 & . & 65 & 3.8 & $\begin{array}{l}-7.60(10.1) \\
-6.80(10.7)^{\mathrm{a}}\end{array}$ & $\begin{array}{l}0.9(2.0) \\
0.5(1.9)^{\text {ad }} \\
\text { (UPE) }\end{array}$ \\
\hline Lam, 1995 & NID-DM & \begin{tabular}{|l|} 
Lovastatin $20-$ \\
$40 \mathrm{mg}$ \\
Placebo \\
\end{tabular} & 34 & 2 & 56 & 56 & 100 & . & 84 & 4.2 & $\begin{array}{l}-1.10(5.7) \\
-1.30(3.6)^{\mathrm{ab}}\end{array}$ & \begin{tabular}{|l|}
$0(0.1)$ \\
$0.25(0.2)^{\mathrm{ab}}$ \\
$(\mathrm{UPE})$ \\
\end{tabular} \\
\hline Lee, 2005 & Controlled HT & \begin{tabular}{|l|} 
Pravastatin \\
$10 \mathrm{mg}$ \\
Placebo \\
\end{tabular} & 61 & 1 & 49 & 68 & 0 & $121 / 73$ & 87 & 3.2 & $\begin{array}{l}13.0(13.3) \\
4.0(12.4)^{\mathrm{a}}\end{array}$ & \begin{tabular}{|l|}
$-673(448)$ \\
$-7(327)^{\mathrm{ab}}$ \\
$(\mathrm{UPE})$ \\
\end{tabular} \\
\hline Lemos, 2013 & CKD & $\begin{array}{l}\text { Rosuvastatin } \\
10 \mathrm{mg} \\
\text { Control } \\
\end{array}$ & 77 & 2 & 58 & 61 & 21 & . & 40 & 3.1 & \begin{tabular}{|l}
$-1.15(6.0)$ \\
$-2.50(5.1) \mathrm{a}$
\end{tabular} & $\begin{array}{l}0.08(0.18) \\
0.23(0.26)^{\mathrm{ad}} \\
(\mathrm{UPE})\end{array}$ \\
\hline Mori, 1992 & NID-DM & $\begin{array}{l}\text { Pravastatin } \\
10 \mathrm{mg} \\
\text { Control }\end{array}$ & 33 & 1 & 63 & 36 & 100 & $134 / 80$ & . & 2.9 & . & $\begin{array}{l}-50.5(54.7) \\
-5.4(71.8)^{\mathrm{a}} \\
(\text { UACR) }\end{array}$ \\
\hline Mou, 2016 & $\begin{array}{l}\text { Chronic glom. } \\
\text { nephritis }\end{array}$ & $\begin{array}{l}\text { Pravastatin } \\
20 \mathrm{mg} \\
\text { Control }\end{array}$ & 48 & 1.8 & 51 & . & 8 & $133 / 75$ & 75 & 3.5 & $\begin{array}{l}-1.08(12.7) \\
-4.33(10.6)^{\mathrm{ab}}\end{array}$ & $\begin{array}{l}-0.33(0.9) \\
-0.27(0.9)^{\mathrm{ab}} \\
(\mathrm{UPE})\end{array}$ \\
\hline $\begin{array}{l}\text { Nanayakkara, } \\
2007 \\
\text { ATIC }\end{array}$ & CKD & $\begin{array}{l}\text { Pravastatin } 40 \\
\text { mg* } \\
\text { Placebo }\end{array}$ & 87 & 2 & 53 & 57 & 0 & $135 / 79$ & 34 & 3.6 & $\begin{array}{l}0(4.3) \\
0.15(4.3)^{\mathrm{ab}}\end{array}$ & \begin{tabular}{|l}
$-0.1(0.8)$ \\
$0.2(0.8)^{\mathrm{a}}$ \\
$\log (\mathrm{UAE})$
\end{tabular} \\
\hline Ohsawa, 2015 & CKD & $\begin{array}{l}\text { Pitavastatin } \\
1-4 \mathrm{mg} \\
\text { Control }\end{array}$ & 28 & 1 & 62 & 71 & 33 & $130 / 78$ & 49 & 3.6 & $\begin{array}{l}-3.50(3.21) \\
-4.20(2.96)^{\mathrm{a}}\end{array}$ & $\begin{array}{l}-244(574) \\
-338(1141)^{\mathrm{a}} \\
(\mathrm{UACR})\end{array}$ \\
\hline $\begin{array}{l}\text { Rahman, } 2008 \\
\text { ALLHAT }\end{array}$ & HT, HCh & \begin{tabular}{|l|} 
Pravastatin \\
$40 \mathrm{mg}$ \\
Control \\
\end{tabular} & 10355 & 6 & 67 & 51 & 35 & $143 / 83$ & 78 & 3.8 & $\begin{array}{l}-1.45(5.9) \\
-1.65(5.9)^{\mathrm{a}}\end{array}$ & . \\
\hline $\begin{array}{l}\text { Rutter, } 2011 \\
\text { PANDA }\end{array}$ & DM2 & \begin{tabular}{|l|} 
Atorvastatin \\
$80 \mathrm{mg}$ \\
Atorvastatin \\
$10 \mathrm{mg}$ \\
\end{tabular} & 119 & 2.5 & 64 & 83 & 100 & . & 67 & 3.1 & $\begin{array}{l}1.0(13.8) \\
-3.0(11.8)^{\mathrm{ab}}\end{array}$ & . \\
\hline Sawara, 2008 & CKD & \begin{tabular}{|l|} 
Rosuvastatin \\
$2.5 \mathrm{mg}$ \\
Control \\
\end{tabular} & 38 & 1 & 65 & 0 & . & $127 / 78$ & 53 & 3.3 & $\begin{array}{l}2.60(12.3) \\
-2.20(10.6)^{\mathrm{a}}\end{array}$ & $\begin{array}{l}-0.04(0.19) \\
0.05(0.24)^{\mathrm{a}} \\
\text { (UPE) }\end{array}$ \\
\hline Scanferla, 1991 & CKD & $\begin{array}{l}\text { Sim/ } \\
\text { pravastatin } \\
10 \mathrm{mg} \\
\text { Control } \\
\end{array}$ & 24 & 1 & 54 & 58 & . & $172 / 106$ & 40 & . & $\begin{array}{l}-1.80(4.2) \\
-3.10(4.2)\end{array}$ & . \\
\hline $\begin{array}{l}\text { Shepherd, } 2007 \\
\text { TNT }\end{array}$ & CAD & \begin{tabular}{|l|} 
Atorvastatin \\
$80 \mathrm{mg}$ \\
Atorvastatin \\
$10 \mathrm{mg}$ \\
\end{tabular} & 10001 & 5 & 61 & 81 & 15 & $131 / 78$ & 65 & 2.5 & $\begin{array}{l}1.5(9.7) \\
0.1(9.7)^{b f}\end{array}$ & . \\
\hline Takazakura, 2015 & DM & \begin{tabular}{|l|} 
Atorvastatin \\
$10 \mathrm{mg}$ \\
Pravastatin \\
$10 \mathrm{mg}$ \\
Control \\
\end{tabular} & 106 & 1 & 62 & 87 & 100 & $129 / 0$ & 64 & 3.0 & $\begin{array}{l}-0.80(11.4) \\
-2.80(10.8) \\
-3.10(9.6)^{\mathrm{a}}\end{array}$ & $\begin{array}{l}-0.2(0.4) \\
-0.1(0.7) \\
0.1(0.5)^{\mathrm{a}} \\
\log (\mathrm{UACR})\end{array}$ \\
\hline $\begin{array}{l}\text { Tonelli, } 2005 \\
\text { PPP ** }\end{array}$ & CAD & $\begin{array}{l}\text { Pravastatin } \\
40 \mathrm{mg} \\
\text { Placebo }\end{array}$ & 18569 & 5 & 58 & 90 & 7 & $133 / 81$ & 73 & 4.2 & $\begin{array}{l}\text { Effect of } \\
\text { pravastatin: } \\
0.10(0.02 ; \\
0.17) \mathrm{mL} / \\
\mathrm{min} / 1.73 \mathrm{~m}^{2 \mathrm{~g}}\end{array}$ & . \\
\hline $\begin{array}{l}\text { Vidt, } 2011 \\
\text { JUPITER }\end{array}$ & $\begin{array}{l}\text { Healthy } \\
\text { population }\end{array}$ & \begin{tabular}{|l|} 
Rosuvastatin \\
$20 \mathrm{mg}$ \\
Placebo \\
\end{tabular} & 16279 & 2.3 & 66 & 62 & 31 & . & 75 & . & $\begin{array}{l}-7.10(11.9) \\
-7.70(11.8)\end{array}$ & . \\
\hline Yakusevich, 2013 & Stroke & \begin{tabular}{|l|} 
Simvastatin \\
$40 \mathrm{mg}$ \\
Control \\
\end{tabular} & 210 & 1 & 66 & 45 & . & . & 76 & 2.2 & $\begin{array}{l}7.05(12.1) \\
1.37(13.8)^{\mathrm{f}}\end{array}$ & . \\
\hline Yasuda, 2004 & CKD & $\begin{array}{l}\text { Fluvastatin } \\
20 \mathrm{mg} \\
\text { Control }\end{array}$ & 80 & 0.9 & 58 & 46 & 43 & $144 / 80$ & 60 & 4.4 & $\begin{array}{l}-8.67(3.9) \\
-6.50(4.0)^{\mathrm{a}}\end{array}$ & \begin{tabular}{|l|}
$0(0.14)$ \\
$0(0.15)^{\mathrm{a}}$ \\
$(\mathrm{UAE})$
\end{tabular} \\
\hline \multicolumn{13}{|l|}{ Continued } \\
\hline
\end{tabular}




\begin{tabular}{|c|c|c|c|c|c|c|c|c|c|c|c|c|}
\hline \multirow[b]{2}{*}{$\begin{array}{l}\text { Author, year } \\
\text { Study name }\end{array}$} & \multirow[b]{2}{*}{ Population } & \multirow[b]{2}{*}{ Intervention } & \multicolumn{8}{|c|}{ Mean baseline characteristics per RCT } & \multicolumn{2}{|c|}{$\begin{array}{l}\text { Outcome [annual change } \\
\text { (SD)] } \\
\text { per intervention }\end{array}$} \\
\hline & & & $\begin{array}{l}\text { Sample } \\
\text { size } \\
(\mathbf{n})\end{array}$ & $\begin{array}{l}\text { Follow- } \\
\text { up } \\
(y)\end{array}$ & $\begin{array}{l}\text { Age } \\
(y)\end{array}$ & $\begin{array}{l}\text { Male } \\
\text { sex } \\
(\%)\end{array}$ & $\begin{array}{l}\text { Diabetes } \\
\text { (\%) }\end{array}$ & $\begin{array}{l}\text { Blood } \\
\text { pressure } \\
(\mathrm{mmHg})\end{array}$ & $\begin{array}{l}\text { eGFR } \\
(\mathrm{mL} / \\
\left.\mathrm{min} / 1.73 \mathrm{~m}^{2}\right)\end{array}$ & $\begin{array}{l}\text { LDL } \\
(\mathrm{mmol} / \mathrm{L})\end{array}$ & eGFR & Proteinuria \\
\hline $\begin{array}{l}\text { De Zeeuw, } 2015 \\
\text { PLANET I }\end{array}$ & DM & $\begin{array}{l}\text { Rosuvastatin } \\
10 \mathrm{mg} \\
\text { Rosuvastatin } \\
40 \mathrm{mg} \\
\text { Atorvastatin } \\
80 \mathrm{mg}\end{array}$ & 325 & 1 & 58 & 70 & 100 & $139 / 79$ & 71 & 3.9 & $\begin{array}{l}-3.70(14.7) \\
-7.29(20.4) \\
-1.61(13.0)\end{array}$ & $\begin{array}{l}2(79) \\
-4(77) \\
-13(57) \\
\text { \%change }\end{array}$ \\
\hline $\begin{array}{l}\text { De Zeeuw, } 2015 \\
\text { PLANET II }\end{array}$ & $\begin{array}{l}\text { Non-DM } \\
\text { proteinuria }\end{array}$ & $\begin{array}{l}\text { Rosuvastatin } \\
10 \mathrm{mg} \\
\text { Rosuvastatin } \\
40 \mathrm{mg} \\
\text { Atorvastatin } \\
80 \mathrm{mg}\end{array}$ & 220 & 1 & 49 & 62 & 0 & $130 / 81$ & 75 & 4.3 & $\begin{array}{l}-2.71(13.3) \\
-3.30(12.5) \\
-1.74(14.2)\end{array}$ & $\begin{array}{l}-6(99) \\
8(75) \\
-24(60)\end{array}$ \\
\hline
\end{tabular}

Table 1. characteristics of included studies. ACS, acute coronary syndrome; ADPKD, autosomal dominant polycystic kidney disease; $\mathrm{CAD}$, coronary artery disease; $\mathrm{CHD}$, coronary heart disease; $\mathrm{CKD}$, chronic kidney disease; eGFR, estimated glomerular filtration rate; eze, ezetimibe; HT, hypertension; MI, myocardial infarction; TIA, transient ischemic attack; (NID-)DM1/DM2, non-insulin dependent diabetes mellitus 1 or 2, LDL, low-density lipoprotein; prot, proteinuria; UACR, urinary albumin-to-creatinine ratio; UAE, urinary albumin excretion; UPE, urinary protein excretion. *Intervention was a combination of statin and vitamin E supplementation. **PPP: Pravastatin Pooling Project, study representing pooled estimates of three RCTs: LIPID, CARE, and WOSCOPS. Individual data on each RCT was not published. abased on eGFR (SD) value at baseline and follow-up. SD of eGFR change was calculated according to the formula provided in the Cochrane Handbook ${ }^{11}$. ${ }^{\mathrm{b}}$ data extracted from figure. ${ }^{\mathrm{c}}$ reported geometric mean was log-transformed to achieve normal distribution with symmetrical SD. dSD acquired by dividing interquartile range by 1.35 . ${ }^{\mathrm{e}}$ no SD or SE reported,

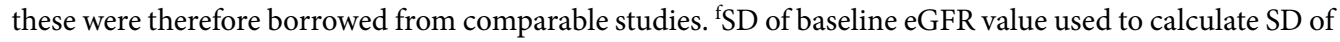
eGFR change. 'only effect of treatment $v s$ control reported.

significant impact on the effect estimates. In post-hoc RCTs $(n=11)$ the beneficial effect on annual kidney function decline of statins $v s$ control was smaller but more precise than in RCTs in which change in eGFR was the primary outcome $(\mathrm{n}=17)$ : 0.55 (95\%-CI $0.19 ; 0.92)$ vs $1.55(95 \%$-CI $0.26 ; 2.85) \mathrm{mL} / \mathrm{min} / 1.73 \mathrm{~m}^{2}$, respectively. In open-label RCTs $(n=17$, mean sample size 4326$)$ the beneficial effect on eGFR decline of statins $v s$ control was stronger than in blinded RCTs ( $\mathrm{n}=13$, mean sample size 1161): 1.25 (95\%-CI 0.08; 2.42) vs 0.23 (95\%-CI 0.11; $0.34) \mathrm{mL} / \mathrm{min} / 1.73 \mathrm{~m}^{2}$, respectively. The funnel plot for eGFR decline was slightly asymmetrical (Supplementary Figure S2), but the Egger's test for small study effects was not significant $(\mathrm{p}=0.3)$.

Pairwise comparison: statins and proteinuria. The two largest RCTs showed that statin treatment $v s$ control did not lower proteinuria: SMD of $0.40(95 \%$-CI $0.18 ; 0.61)$ and $0.18(95 \%$-CI $0.04 ; 0.32)$, respectively ${ }^{32,63}$. In a meta-analysis, statin use compared to control showed a significant reduction of proteinuria with an SMD $-0.58(95 \%$-CI $-0.88 ;-0.29)$ (Fig. 3). However, the funnel plot of the effect of statins on proteinuria suggested publication bias (Supplementary Figure S3) and the Egger's test was significant $(\mathrm{p}<0.001)$.

Network meta-analysis. Figure 4A shows the network plot of different statin treatments for change in eGFR. Each connection was formed by maximally 4 RCTs. We found no evidence for inconsistency in the network for eGFR decline and proteinuria using global tests ( $p$-value for inconsistency 0.8 ) or local tests $(p>0.3$ for all loops). We found that almost all statins performed better than control (Fig. 5). The most beneficial effect on eGFR decline was caused by fluvastatin $20 \mathrm{mg}$ /ezetimibe $10 \mathrm{mg}$, rosuvastatin $20 \mathrm{mg}$ /ezetimibe $10 \mathrm{mg}$, pravastatin $10-20 \mathrm{mg}$, and atorvastatin $40-80$ and $10<40 \mathrm{mg}$. However, point estimates had broad $95 \%$-confidence intervals and prediction intervals. Except for combined fluvastatin $20 \mathrm{mg}$ /ezetimibe $10 \mathrm{mg}$ and atorvastatin $40-80 \mathrm{mg}$, all 95\%-confidence intervals crossed the line of no effect.

Figure 4B shows the network plot for all statin treatments regarding proteinuria. For proteinuria, no single RCT compared the combination therapy simvastatin/ezetimibe. Globally, there was no evidence for inconsistency (p-value 0.8 ). However, using local tests, there were 2 inconsistent loops: control, atorvastatin $40-80 \mathrm{mg}$, rosuvastatin $2-10 \mathrm{mg}(\mathrm{p}=0.04)$ and control, simvastatin $10-40 \mathrm{mg}$, lovastatin $20-40 \mathrm{mg}(\mathrm{p}=0.03)$. The inconsistencies between direct and indirect effects were introduced by the relatively large effect estimates of small studies $(\mathrm{n}<60)$. The most efficacious treatments regarding proteinuria were fluvastatin $20 \mathrm{mg} /$ ezetimibe $10 \mathrm{mg}$, atorvastatin $40-80 \mathrm{mg}$, and rosuvastatin $20 \mathrm{mg}$ /ezetimibe $10 \mathrm{mg}$ (Fig. 6).

Finally, SUCRA analysis showed that control treatment had the lowest SUCRA. Fluvastatin $20 \mathrm{mg} / \mathrm{ezetimibe}$ $10 \mathrm{mg}$ had the highest SUCRA value for eGFR decline (99\%) and fluvastatin $20 \mathrm{mg} /$ ezetimibe $10 \mathrm{mg}(86 \%)$ as well as atorvastatin $40-80 \mathrm{mg}(78 \%)$ had the highest SUCRA value for change in proteinuria (Fig. 7 ).

Sensitivity analyses. Since we included RCTs with seven different types of statin treatments with one or more different dosages, networks of subgroups had only few closed loops. Therefore, estimates were based mostly on either direct or indirect evidence, but not on mixed evidence. Nonetheless, we repeated the network meta-analysis for eGFR decline excluding RCTs with a sample size $<100(n=16)$, excluding open-label RCTs 


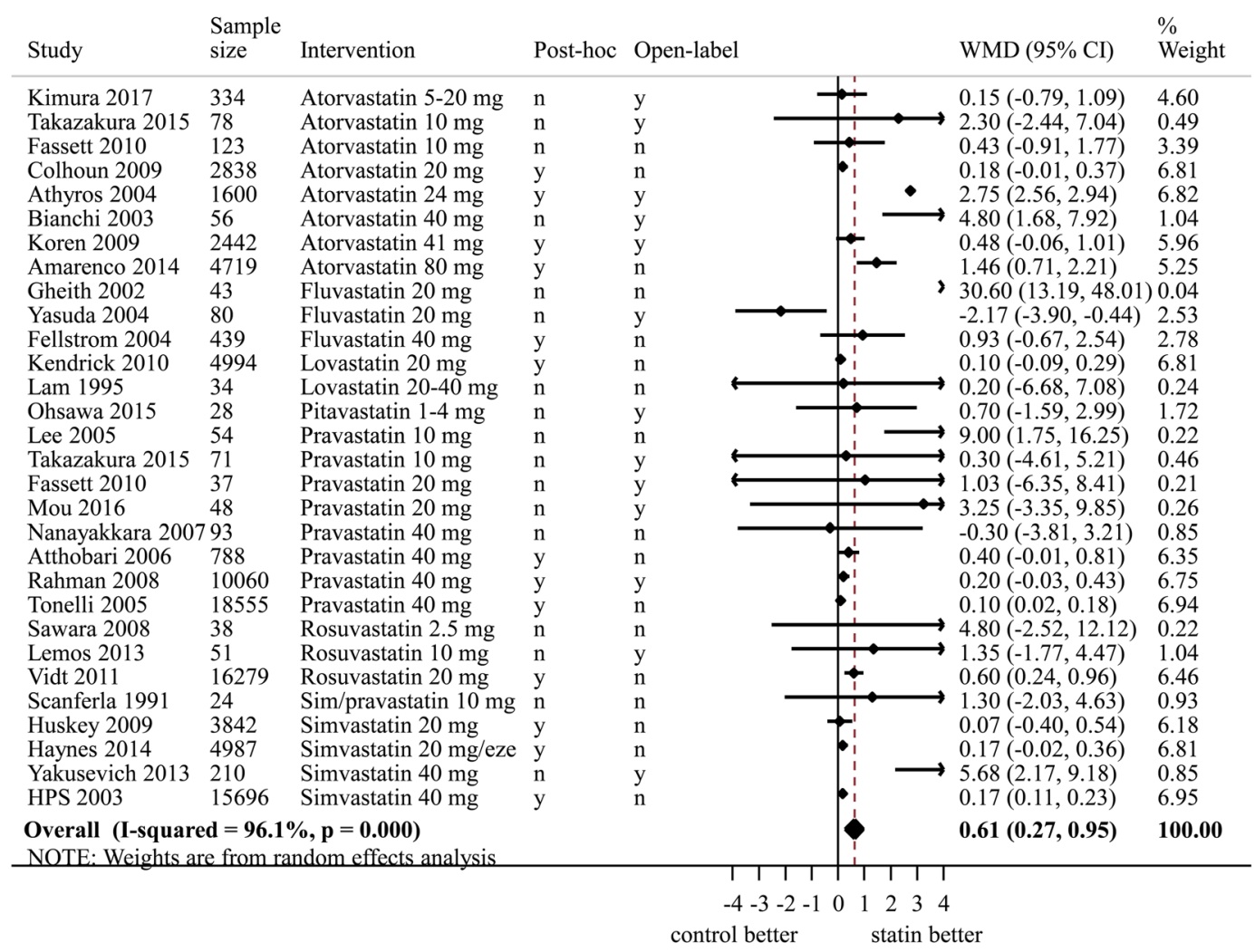

Change in annual eGFR decline, $\mathrm{mL} / \mathrm{min} / 1.73 \mathrm{~m}^{2}$

Figure 2. Pairwise random effects meta-analysis of randomized controlled trials investigating the effect of statin therapy versus control on the rate of annual eGFR decline. Positive values mean slower eGFR decline for statin users $v s$ non-users, thus favouring statin use. In this forest plot, only 30 RCTs are included that compare a statin intervention vs non-statin control intervention. We thus excluded 13 RCTs that reported only the outcome proteinuria $(n=3)$, or that compared two statin interventions $(n=10)$. eGFR, estimated glomerular filtration rate; eze, ezetimibe $10 \mathrm{mg}$; CI, confidence interval; WMD, weighted mean difference; n, no; y, yes.

$(n=17)$, or excluding post-hoc analyses $(n=20)$. Although effect estimates and rankings of individual treatments were variable across the analyses, in general atorvastatin $40-80 \mathrm{mg}$, fluvastatin $20 \mathrm{mg} / \mathrm{ezetimibe} 10 \mathrm{mg}$, pravastatin $10-20 \mathrm{mg}$, simvastatin $10-40 \mathrm{mg}$, and fluvastatin $20 \mathrm{mg}$ were the most effective treatments with regard to eGFR decline. However, 95\%-confidence intervals had substantial overlap, and individual treatments were rarely statistically significantly different from control. Since only a small number of RCTs with small sample sizes studied the effect of statins on proteinuria, we could not perform the aforementioned sensitivity analyses.

\section{Discussion}

In this network meta-analysis, we showed that there are no substantial differences in the efficacy of seven different statins and dosages, with or without ezetimibe, regarding slowing down eGFR decline or reducing proteinuria. If anything, the combination of fluvastatin $20 \mathrm{mg}$ /ezetimibe $10 \mathrm{mg}$ and atorvastatin $40-80 \mathrm{mg}$ most consistently had the strongest beneficial effect on both renal endpoints, but the differences between treatments were small and confidence intervals were wide. In the pairwise meta-analysis we showed that use of statins lowered the rate of annual kidney function decline by $0.61 \mathrm{~mL} / \mathrm{min} / 1.73 \mathrm{~m}^{2}$ and reduced the amount of proteinuria by -0.58 (95\%-CI $-0.88 ;-0.29)$ standard deviations per year.

Our results are in line with a recent meta-analysis $\mathrm{Su}$ et al. which reported that statins compared to control led to a $0.41(95 \%$-CI $0.11 ; 0.70) \mathrm{mL} / \mathrm{min} / 1.73 \mathrm{~m}^{2}$ slower annual eGFR decline and a reduction of -0.65 $(95 \%-C I-0.94 ;-0.37)$ standard deviations in proteinuria ${ }^{8}$. The small difference in outcomes between the present study and $\mathrm{Su}$ et al. are explained by different inclusion criteria. In contrast to the study of Su et al., we included three RCTs investigating combinations of statins plus ezetimibe. Including also treatments combining statins with ezetimibe, results in a more complete review of existing literature on lipid-lowering therapy by statins. As a consequence we incorporated in our meta-analysis three extra RCTs, including the SHARP trial $(\mathrm{n}=5037)$. Furthermore, we excluded RCTs with a short follow-up ( $<12$ months) or less than 10 patients per study arm, of which Su et al. included 19 RCTs. Finally, we found that the beneficial effect of statins on eGFR decline was weaker in RCTs with a higher mean systolic blood pressure. Systolic blood pressure explained $40 \%$ of the between-study variance. Taken together, these results suggest that a high systolic blood pressure modifies the effect of statins on eGFR decline. Hypertension is most likely a stronger risk factor for kidney function decline compared to 


\begin{tabular}{|c|c|c|c|c|c|c|c|}
\hline Study & $\begin{array}{l}\text { Sample } \\
\text { size }\end{array}$ & Intervention & Post-hoc & Open-label & & $\operatorname{SMD}(95 \% \mathrm{CI})$ & $\begin{array}{l}\% \\
\text { Weight }\end{array}$ \\
\hline Kimura 2017 & 334 & Atorvastatin $5-20 \mathrm{mg}$ & $\mathrm{n}$ & $\mathrm{y}$ & $\leftarrow$ & $0.40(0.18,0.61)$ & 6.37 \\
\hline Dalla Nora 2003 & 25 & Atorvastatin $10 \mathrm{mg}$ & $\mathrm{n}$ & $\mathrm{n}$ & & $-2.11(-3.10,-1.11)$ & 3.75 \\
\hline Fassett 2010 & 123 & Atorvastatin $10 \mathrm{mg}$ & $\mathrm{n}$ & $\mathrm{n}$ & & $-0.32(-0.67,0.04)$ & 6.01 \\
\hline Takazakura 2015 & 78 & Atorvastatin $10 \mathrm{mg}$ & $\mathrm{n}$ & $\mathrm{y}$ & & $-0.64(-1.10,-0.18)$ & 5.68 \\
\hline Bianchi 2003 & 56 & Atorvastatin $40 \mathrm{mg}$ & $\mathrm{n}$ & $\mathrm{y}$ & & $-1.61(-2.21,-1.00)$ & 5.15 \\
\hline Gheith 2002 & 43 & Fluvastatin $20 \mathrm{mg}$ & $\mathrm{n}$ & $\mathrm{n}$ & & $-1.70(-2.41,-1.00)$ & 4.78 \\
\hline Yasuda 2004 & 80 & Fluvastatin $20 \mathrm{mg}$ & $\mathrm{n}$ & $\mathrm{y}$ & & $0.00(-0.44,0.44)$ & 5.75 \\
\hline Lam 1995 & 34 & Lovastatin $20-40 \mathrm{mg}$ & $\mathrm{n}$ & $\mathrm{n}$ & & $-1.31(-2.06,-0.57)$ & 4.62 \\
\hline Ohsawa 2015 & 28 & Pitavastatin $1-4 \mathrm{mg}$ & $\mathrm{n}$ & $\mathrm{y}$ & & $0.10(-0.64,0.85)$ & 4.64 \\
\hline Lee 2005 & 54 & Pravastatin $10 \mathrm{mg}$ & $\mathrm{n}$ & $\mathrm{n}$ & & $-1.78(-2.44,-1.13)$ & 4.96 \\
\hline Mori 1992 & 33 & Pravastatin $10 \mathrm{mg}$ & $\mathrm{n}$ & $\mathrm{y}$ & & $-0.72(-1.42,-0.01)$ & 4.76 \\
\hline Takazakura 2015 & 71 & Pravastatin $10 \mathrm{mg}$ & $\mathrm{n}$ & $\mathrm{y}$ & & $-0.33(-0.81,0.15)$ & 5.61 \\
\hline Fassett 2010 & 37 & Pravastatin $20 \mathrm{mg}$ & $\mathrm{n}$ & $\mathrm{y}$ & & $-0.19(-0.84,0.46)$ & 4.99 \\
\hline Mou 2016 & 48 & Pravastatin $20 \mathrm{mg}$ & $\mathrm{n}$ & $\mathrm{y}$ & & $-0.66(-1.24,-0.07)$ & 5.24 \\
\hline Atthobari 2006 & 788 & Pravastatin $40 \mathrm{mg}$ & $\mathrm{y}$ & $\mathrm{n}$ & & $0.18(0.04,0.32)$ & 6.50 \\
\hline Nanayakkara 2007 & 93 & Pravastatin $40 \mathrm{mg}$ & $\mathrm{n}$ & $\mathrm{n}$ & & $-0.38(-0.79,0.03)$ & 5.84 \\
\hline Sawara 2008 & 38 & Rosuvastatin $2.5^{\circ} \mathrm{mg}$ & $\mathrm{n}$ & $\mathrm{n}$ & & $-0.43(-1.08,0.22)$ & 4.98 \\
\hline Lemos 2013 & 51 & Rosuvastatin $10 \mathrm{mg}$ & $\mathrm{n}$ & $\mathrm{y}$ & & $-0.64(-1.20,-0.07)$ & 5.29 \\
\hline Fried 2001 & 39 & Simvastatin $10 \mathrm{mg}$ & $\mathrm{n}$ & $\mathrm{n}$ & & $-0.09(-0.72,0.54)$ & 5.06 \\
\hline \multicolumn{6}{|c|}{$\begin{array}{l}\text { Overall (I-squared }=\mathbf{8 8 . 1} \%, \mathbf{p}=\mathbf{0 . 0 0 0} \text { ) } \\
\text { NOTE: Weights are from random effects analysis }\end{array}$} & $-0.58(-0.88,-0.29)$ & 100.00 \\
\hline
\end{tabular}

Figure 3. Pairwise random effects meta-analysis of randomized controlled trials investigating the effect of statin therapy versus control on the rate of annual change in proteinuria. Negative values mean a decrease in proteinuria for statin users $v s$ non-users, thus favouring statin use. Effects expressed as SMD (standardized mean difference). In this forest plot, only 19 RCTs are included that compare a statin intervention vs non-statin control intervention. We thus excluded 24 RCTs that reported only the outcome proteinuria $(n=18)$, or that compared two statin interventions $(n=6)$. CI, confidence interval; SMD, standardized mean difference; $n$, no; $y$, yes.

hypercholesteremia. Therefore, we speculate that the positive effect of statins on kidney function decline is overwhelmed in the presence of high blood pressure.

In our network meta-analysis, we specifically investigated the efficacy of individual statins and different dosages, using both direct and indirect evidence. We showed that each different statin compared to placebo had a beneficial effect on the annual eGFR decline and reduced proteinuria. However, confidence intervals were broad for individual treatment comparisons in our network, due to the small number of RCTs contributing to each comparison. Su et al. showed in subgroup analyses the strongest beneficial effect on change in eGFR decline for atorvastatin, fluvastatin, and rosuvastatin ${ }^{8}$. However, they pooled for each statin all dosages. The validity of these comparisons may be limited, considering the clear differential effects of different dosages ${ }^{8,66}$.

We showed that fluvastatin $20 \mathrm{mg}$ /ezetimibe $10 \mathrm{mg}$ was the most efficacious treatment regarding both renal outcomes. However, this result was strongly influenced by the study of Kinouchi et al., comprising 54 patients, reporting an annual eGFR decline of $-4.1 \mathrm{~mL} / \mathrm{min} / 1.73 \mathrm{~m}^{2}$ in patients treated with fluvastatin $20 \mathrm{mg}$ compared to an annual eGFR increase of $4.1 \mathrm{~mL} / \mathrm{min} / 1.73 \mathrm{~m}^{2}$ in patients treated with fluvastatin $20 \mathrm{mg} /$ ezetimibe $10 \mathrm{mg}^{46}$. Since the average annual eGFR decline in adults with a history of cardiovascular disease is about $2 \mathrm{~mL} /$ $\mathrm{min} / 1.73 \mathrm{~m}^{2}$, the reported effect of Kinouchi et al. of $8.2 \mathrm{~mL} / \mathrm{min} / 1.73 \mathrm{~m}^{2}$ is large, and should be interpreted with caution $^{67}$. We found that the second most efficacious statin on both renal endpoints was high dose atorvastatin, which improved the annual eGFR decline by $1.70(95 \%$-CI $0.70 ; 2.70) \mathrm{mL} / \mathrm{min} / 1.73 \mathrm{~m}^{2}$ and reduced proteinuria by $1.14(95 \%$-CI $0.28 ; 2.00)$ standard deviations, compared to control.

Statins included in the present study reduced LDL levels on average by $27 \%$, which is in line with a previous meta-analysis showing an LDL-lowering effect for all statins ${ }^{66}$. However, there is no clear evidence that high LDL itself increases CKD risk ${ }^{68}$. Statins also may have pleiotropic effects favourable for reducing CKD progression, such as lowering oxidative stress, reducing inflammation, and stabilizing atherosclerotic plaques ${ }^{7,69}$. Hence, current guidelines recommend a statin for patients at risk for CKD, independent of LDL levels ${ }^{9,70}$.

The main strength of the current study is that we performed a network meta-analysis, in addition to a pairwise meta-analysis, to investigate differential effects of different statins with or without ezetimibe. We only included RCTs because they are more likely to provide unbiased information. We excluded small trials ( $<10$ patients per arm) since they are more susceptible to publication bias.

This network meta-analysis has several limitations. First, heterogeneity was high $\left(\mathrm{I}^{2}=96 \%\right)$ owing to variation of the included patient populations across RCTs, differences in blinding methods, randomization procedures, sample size, and variability in primary endpoints. The $\mathrm{I}^{2}$ statistic represents statistical heterogeneity, rather than clinically relevant heterogeneity, and is most strongly affected by the sample size of the individual studies. Upon increasing precision (sample size) of studies within a meta-analysis, the $\mathrm{I}^{2}$ statistic rapidly approaches $100 \%{ }^{71}$. Deciding whether it is valid to pool studies, should be based on the clinical relevance of any present heterogeneity, rather than solely on the $\mathrm{I}^{2}$ statistic ${ }^{71}$. We used random effects models to take heterogeneity into account. Second, we found an asymmetric funnel plot regarding proteinuria, which may be an indication of publication bias. On the other hand, larger compared to smaller RCTs showed a weak but opposite effect. Thus, the asymmetry may 


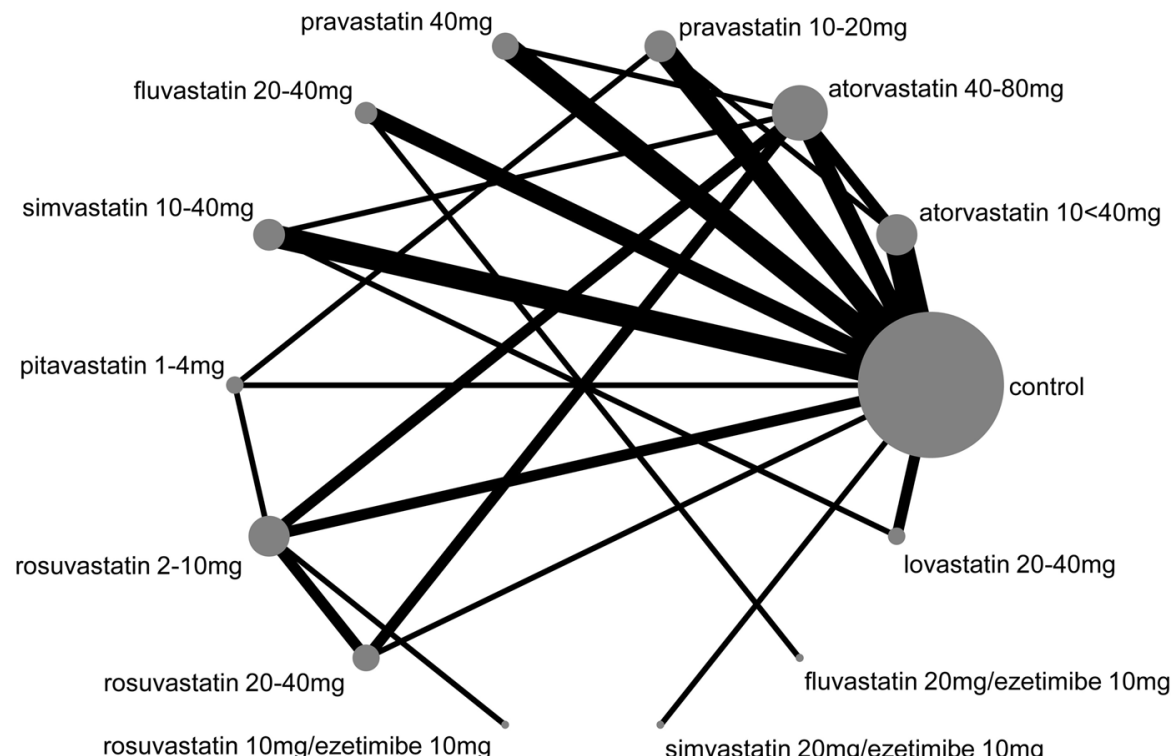

rosuvastatin $10 \mathrm{mg} / \mathrm{ezetimibe} 10 \mathrm{mg}$

simvastatin 20mg/ezetimibe $10 \mathrm{mg}$

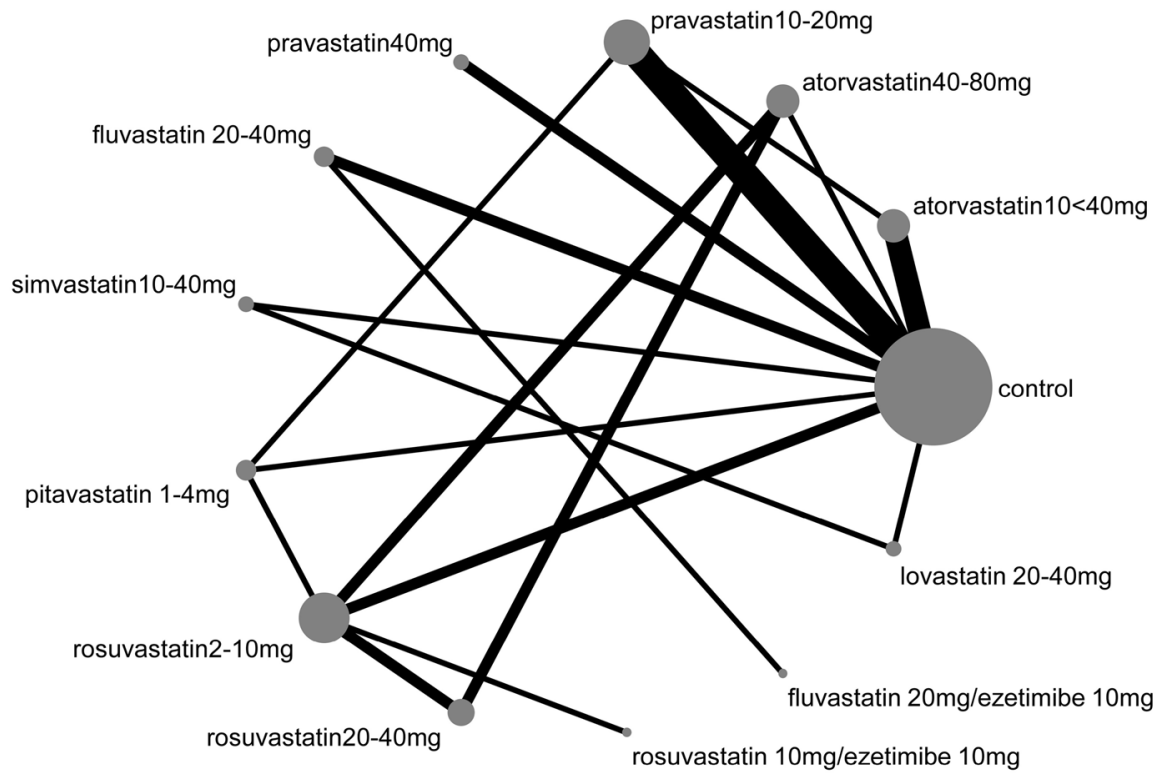

Figure 4. Network plots for the outcome eGFR decline (A) and proteinuria (B). The size of the nodes represents the number of RCTs for each treatment (ranging from 1 to $30 ; 30$ for control intervention). The width of the connections represents the number of RCTs for each individual comparison (ranging from 1 to 5). eGFR, estimated glomerular filtration rate; RCT, randomized controlled trial.

also be the consequence of inclusion of smaller RCTs with lower quality. Therefore, we cannot rule out that the beneficial effect of statins on proteinuria is an overestimation. Additionally, there were relatively few RCTs investigating the effect of statins on proteinuria, and most of them were small (sample size $<100$ ). Small studies therefore had a large impact on the network meta-analysis estimates, introducing inconsistencies especially in loops comprising small numbers of RCTs. The advantage of a network analysis is that it takes both direct and indirect effects into account, reducing the impact of single studies with a small sample size. For the outcome eGFR decline, the sample sizes of the included RCTs were large (24 RCTs with $n>100)$ which improved precision and reduced potential publication bias. The much smaller effect of statins compared to control in double blind compared to open-label RCTs may suggest bias due to the lack of blinding in the open-label RCTs. Since 17 out of 30 RCTs were open-label, we may have overestimated the beneficial effect on eGFR decline of statins compared to control. Third, due to the low number of RCTs contributing to each connection in the network meta-analyses, there was insufficient power to detect differences between statins. Fourth, a large number of the included RCTs used the MDRD formula to estimate eGFR, which is known to underestimate the true eGFR for values reported higher than $60 \mathrm{~mL} / \mathrm{min} / 1.73 \mathrm{~m}^{272}$. If anything, this may have underestimated the beneficial effect of statin use compared to control in studies with a mean eGFR higher than $60 \mathrm{~mL} / \mathrm{min} / 1.73 \mathrm{~m}^{2}$. 
Reduction of annual eGFR decline for different statins compared to control

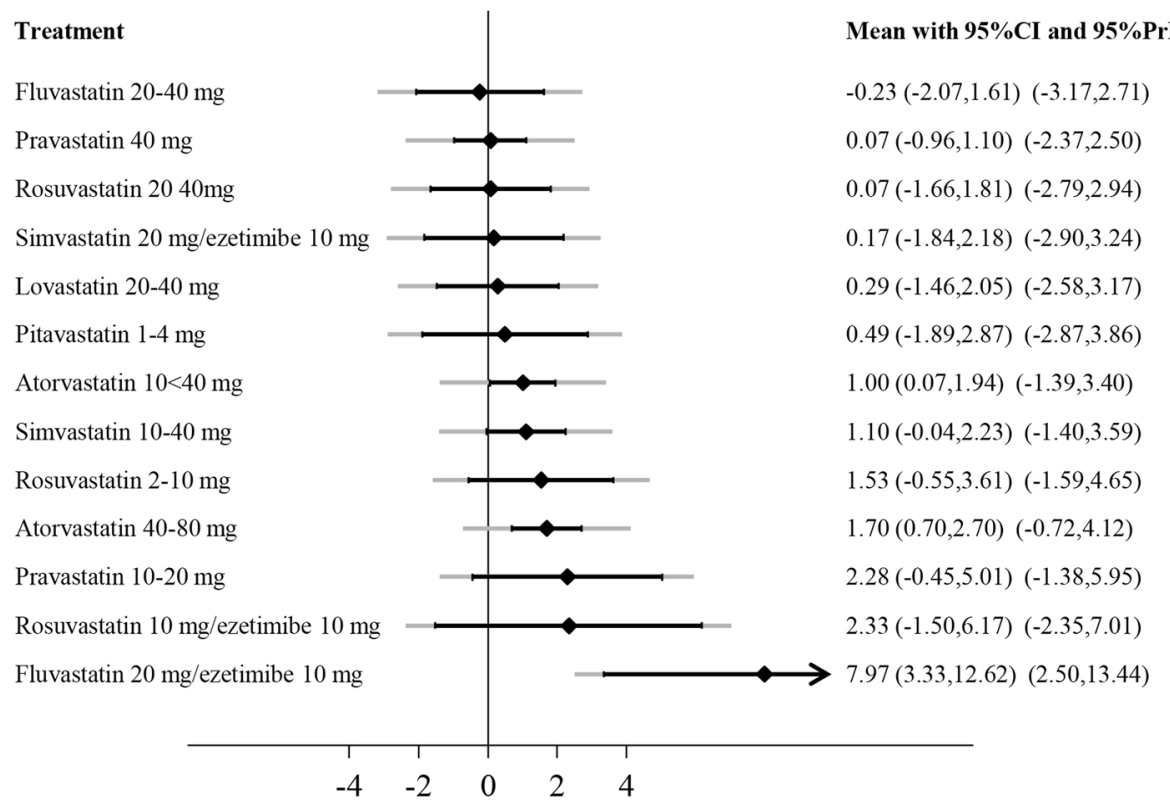

Figure 5. Effect of different statins compared to control treatment on annual eGFR decline. Effects are presented as weighted mean differences. Positive values represent a slower eGFR decline. Black lines around point estimates reflect $95 \%$-confidence intervals and grey lines reflect prediction intervals. Prediction intervals represent the expected range of true effects of (future) similar studies and is suitable to assess the variability of an effect across different settings. CI, confidence interval; eGFR, estimated glomerular filtration rate; PrI, prediction interval.

\section{Annual change in proteinuria for different statins compared to control}

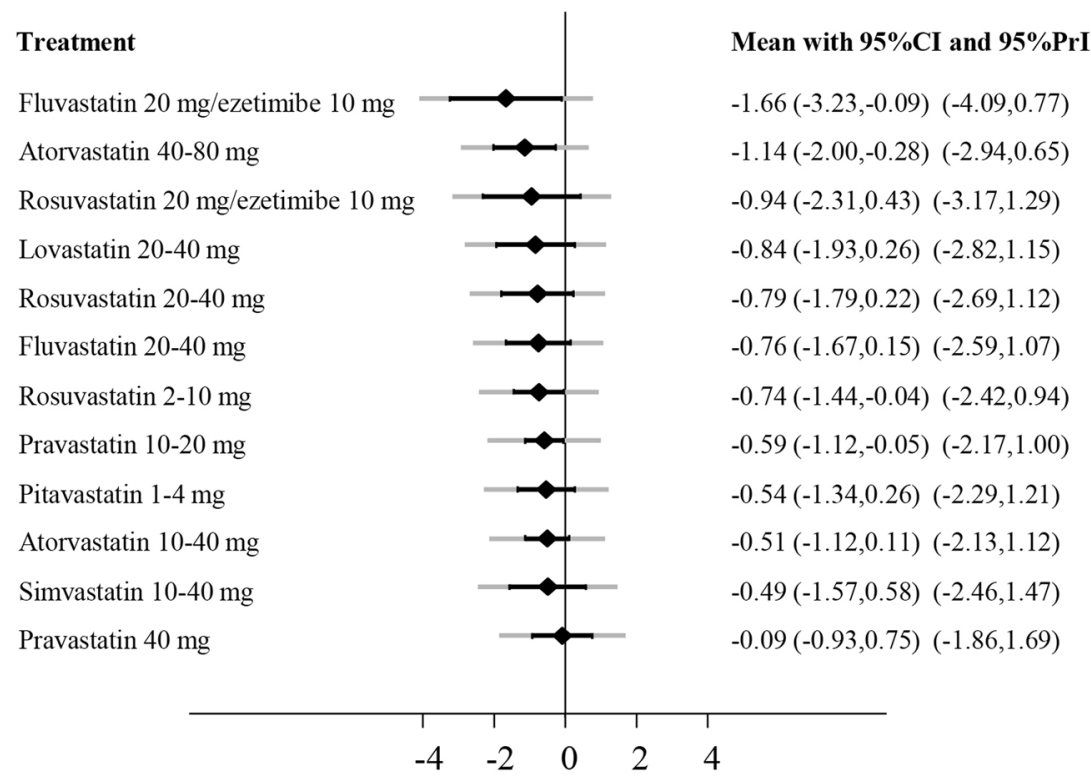

Figure 6. Effect of different statins compared to control treatment on annual change in proteinuria. Effects are presented as standardized mean differences (SMD). Negative values represent a reduction of proteinuria. Black lines around point estimates reflect $95 \%$-confidence intervals and grey lines reflect prediction intervals. Prediction intervals represent the expected range of true effects in (future) similar studies and is suitable to assess the variability of effect across different settings. CI, confidence interval; eGFR, estimated glomerular filtration rate; PrI, prediction interval. 


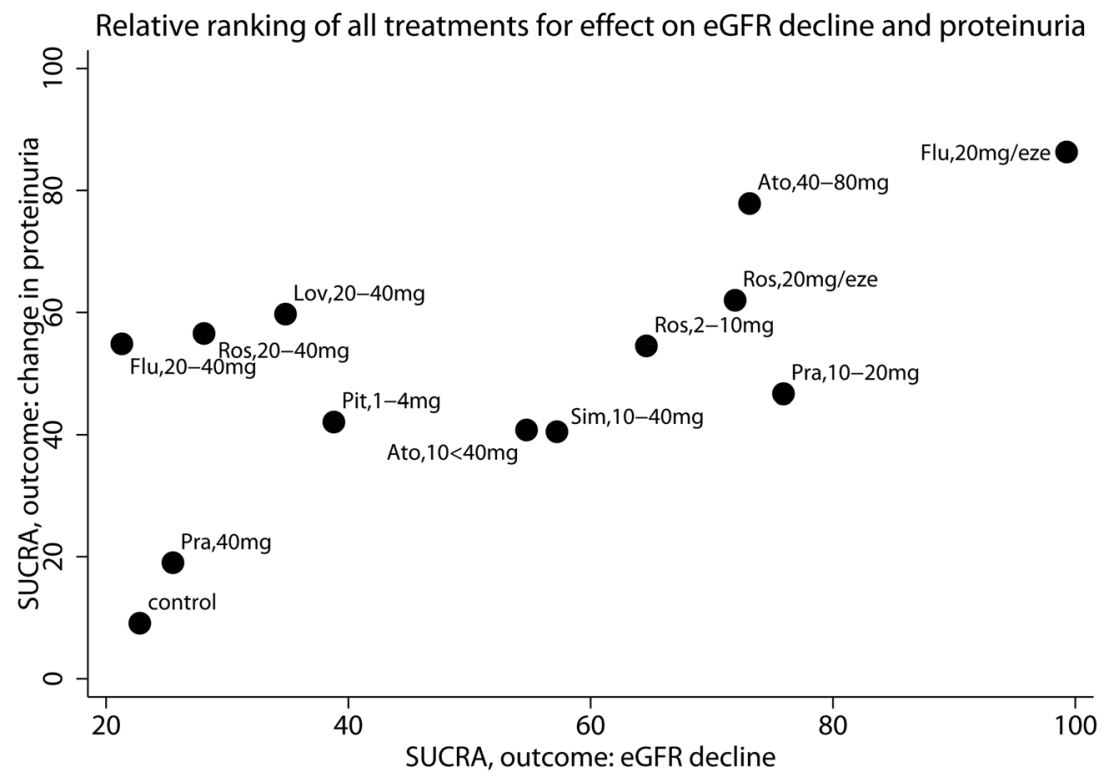

Figure 7. Each dot represents the SUCRA value of each treatment. The SUCRA takes into account for every treatment the cumulative probabilities of all possible rankings. If a treatment always ranks first or last, the SUCRA is $100 \%$ or $0 \%$, respectively. The horizontal axis shows SUCRA values with regards to the outcome eGFR decline, the vertical axis shows the SUCRA for the outcome proteinuria. Ato, atorvastatin; eze, ezetimibe $10 \mathrm{mg}$; Flu, fluvastatin; Lov, lovastatin; Pit, pitavastatin; Pra, pravastatin; Ros, rosuvastatin; Sim, simvastatin; eGFR, estimated glomerular filtration rate; SUCRA, surface under the cumulative ranking curve.

In conclusion, we found a beneficial effect of different statins, with or without ezetimibe, compared to control on progression of eGFR decline, and possibly proteinuria. Due to the imprecision of individual comparisons, results were inconclusive as to which statin performs best with regard to renal outcome.

Received: 22 May 2019; Accepted: 25 October 2019;

Published online: 12 November 2019

\section{References}

1. El Nahas, A. M. \& Bello, A. K. Chronic kidney disease: the global challenge. The Lancet 365, 331-340 (2005).

2. Brück, K. et al. CKD Prevalence Varies across the European General Population. J. Am. Soc. Nephrol. (2015).

3. Hoogeveen, E. K. et al. Kidney function and specific mortality in 60-80 years old post-myocardial infarction patients: A 10-year follow-up study. PLoS One 12, e0171868 (2017).

4. Obermayr, R. P. et al. Predictors of new-onset decline in kidney function in a general middle-european population. Nephrol. Dial. Transplant. 23, 1265-1273 (2008).

5. Hobeika, L., Hunt, K. J., Neely, B. A. \& Arthur, J. M. Comparison of the Rate of Renal Function Decline in NonProteinuric Patients With and Without Diabetes. Am. J. Med. Sci. 350, 447-452 (2015).

6. Kidney Disease. Improving Global Outcomes (KDIGO) Lipid Work Group. KDIGO Clinical Practice Guideline for Lipid Management in Chronic Kidney Disease. Kidney Int. Suppl. 3, 259-305 (2013).

7. Catapano, A. L. et al. 2016 ESC/EAS Guidelines for the Management of Dyslipidaemias: The Task Force for the Management of Dyslipidaemias of the European Society of Cardiology (ESC) and European Atherosclerosis Society (EAS) Developed with the special contribution of the European Association for Cardiovascular Prevention \&amp; Rehabilitation (EACPR). Atherosclerosis 253, 281-344 (2016).

8. Su, X. et al. Effect of Statins on Kidney Disease Outcomes: A Systematic Review and Meta-analysis. Am. J. Kidney Dis. 67, 881-892 (2016).

9. Oesterle, A., Laufs, U. \& Liao, J. K. Pleiotropic Effects of Statins on the Cardiovascular System. Circ. Res. 120, 229-243 (2017).

10. Hutton, B. et al. The prisma extension statement for reporting of systematic reviews incorporating network meta-analyses of health care interventions: Checklist and explanations. Ann. Intern. Med. 162, 777-784 (2015).

11. Booth, A. PROSPERO's progress and activities 2012/13. Systematic Reviews 2, 111 (2013).

12. Higgins, J. P. T. \& Green, S. Cochrane Handbook for Systematic Reviews of Interventions Version 5.1.0 [updated March 2011]. (Part 3, chapter 16.1.3.2. The Cochrane Collaboration, 2011).

13. Fassett, R. G., Coombes, J. S., Packham, D., Fairley, K. F. \& Kincaid-Smith, P. Effect of pravastatin on kidney function and urinary protein excretion in autosomal dominant polycystic kidney disease. Scand. J. Urol. Nephrol. 44, 56-61 (2010).

14. Fassett, R. G., Robertson, I. K., Ball, M. J., Geraghty, D. P. \& Coombes, J. S. Effect of atorvastatin on kidney function in chronic kidney disease: a randomised double-blind placebo-controlled trial. Atherosclerosis 213, 218-224 (2010).

15. Vidt, D. G., Ridker, P. M., Monyak, J. T., Schreiber, M. J. \& Cressman, M. D. Longitudinal assessment of estimated glomerular filtration rate in apparently healthy adults: a post hoc analysis from the JUPITER study (justification for the use of statins in prevention: an intervention trial evaluating rosuvastatin). Clin. Ther. 33, 717-725 (2011).

16. Hoogeveen, E. K. et al. Effect of omega-3 fatty acids on kidney function after myocardial infarction: the Alpha Omega Trial. Clin. J. Am. Soc. Nephrol. 9, 1676-1683 (2014).

17. van den Brand, J. A. J. G. et al. Predicting kidney failure from longitudinal kidney function trajectory: A comparison of models. PLoS One 14, e0216559 (2019).

18. Higgins, J. P. T. et al. The Cochrane Collaboration's tool for assessing risk of bias in randomised trials. The BMJ 343, d5928 (2011).

19. Higgins, J. P., Thompson, S. G., Deeks, J. J. \& Altman, D. G. Measuring inconsistency in meta-analyses. BMJ 327, 557-560 (2003). 
20. Egger, M., Davey Smith, G., Schneider, M. \& Minder, C. Bias in meta-analysis detected by a simple, graphical test. BMJ 315, 629-634 (1997).

21. Noordzij, M., Hooft, L., Dekker, F. W., Zoccali, C. \& Jager, K. J. Systematic reviews and meta-analyses: when they are useful and when to be careful. Kidney Int. 76, 1130-1136 (2009).

22. Rouse, B., Chaimani, A. \& Li, T. Network meta-analysis: an introduction for clinicians. Intern. Emerg. Med. 12, 103-111 (2017).

23. IntHout, J., Ioannidis, J. P. A., Rovers, M. M. \& Goeman, J. J. Plea for routinely presenting prediction intervals in meta-analysis. BMJ Open 6, e010247 (2016).

24. Mbuagbaw, L. et al. Approaches to interpreting and choosing the best treatments in network meta-analyses. Systematic Reviews 6 , 79 (2017).

25. Salanti, G., Ades, A. E. \& Ioannidis, J. P. A. Graphical methods and numerical summaries for presenting results from multipletreatment meta-analysis: an overview and tutorial. J. Clin. Epidemiol. 64, 163-171 (2011).

26. Chaimani, A., Higgins, J. P. T., Mavridis, D., Spyridonos, P. \& Salanti, G. Graphical Tools for Network Meta-Analysis in STATA. PLoS One 8, e76654 (2013).

27. Yakusevich, V. V., Malygin, A. Y. \& Kabanov, A. V. Effect of simvastatin on the prognosis and the changes of the clinical status in patients with acute ischemic stroke. The results of the 12 month randomized, open comparative study. [Russian]. Rational Pharmacotherapy in Cardiology 9, 379-385 (2013).

28. Mori, Y. \& Tsuruoka, A. Effect of pravastatin on microalbuminuria in patients with non-insulin-dependent diabetes mellitus. Journal of the japan diabetes society 35, 265-268 (1992).

29. Abe, M., Maruyama, N., Maruyama, T., Okada, K. \& Soma, M. A Trial of Pitavastatin Versus Rosuvastatin for Dyslipidemia in Chronic Kidney Disease. J Atheroscler. Thromb 22, 1235-1247 (2015).

30. Amarenco, P. et al. Effect of high-dose atorvastatin on renal function in subjects with stroke or transient ischemic attack in the SPARCL trial. Stroke 45, 2974-2982 (2014).

31. Athyros, V. G. et al. The effect of statins versus untreated dyslipidaemia on renal function in patients with coronary heart disease. A subgroup analysis of the Greek atorvastatin and coronary heart disease evaluation (GREACE) study. J. Clin. Pathol. 57, 728-734 (2004).

32. Atthobari, J. et al. The effect of statins on urinary albumin excretion and glomerular filtration rate: results from both a randomized clinical trial and an observational cohort study. Nephrol. Dial. Transplant. 21, 3106-3114 (2006).

33. Bianchi, S., Bigazzi, R., Caiazza, A. \& Campese, V. M. A controlled, prospective study of the effects of atorvastatin on proteinuria and progression of kidney disease. Am. J. Kidney Dis. 41, 565-570 (2003).

34. Castelao, A. M. et al. HMGCoA reductase inhibitors lovastatin and simvastatin in the treatment of hypercholesterolemia after renal transplantation. Transplant. Proc. 25, 1043-1046 (1993).

35. Colhoun, H. M. et al. Effects of atorvastatin on kidney outcomes and cardiovascular disease in patients with diabetes: an analysis from the Collaborative Atorvastatin Diabetes Study (CARDS). Am. J. Kidney Dis. 54, 810-819 (2009).

36. de, Z. D. et al. Renal effects of atorvastatin and rosuvastatin in patients with diabetes who have progressive renal disease (PLANET I): a randomised clinical trial. Lancet Diabetes Endocrinol 3, 181-190 (2015).

37. Deedwania, P. C., Stone, P. H., Fayyad, R. S., Laskey, R. E. \& Wilson, D. J. Improvement in Renal Function and Reduction in Serum Uric Acid with Intensive Statin Therapy in Older Patients: A Post Hoc Analysis of the SAGE Trial. Drugs Aging 32, 1055-1065 (2015).

38. Fellstrom, B. et al. Effect of fluvastatin on renal end points in the Assessment of Lescol in Renal Transplant (ALERT) trial. Kidney Int. 66, 1549-1555 (2004).

39. Gheith, O. A. et al. Impact of treatment of dyslipidemia on renal function, fat deposits and scarring in patients with persistent nephrotic syndrome. Nephron 91, 612-619 (2002).

40. Haynes, R. et al. Effects of lowering LDL cholesterol on progression of kidney disease. J. Am. Soc. Nephrol. 25, 1825-1833 (2014).

41. Heart Protection Study. MRC/BHF Heart Protection Study of cholesterol-lowering with simvastatin in 5963 people with diabetes: a randomised placebo-controlled trial. The Lancet 361, 2005-2016 (2003).

42. Holme, I. et al. Cardiovascular outcomes and their relationships to lipoprotein components in patients with and without chronic kidney disease: results from the IDEAL trial. J. Intern. Med. 267, 567-575 (2010).

43. Huskey, J. et al. Effect of simvastatin on kidney function loss in patients with coronary heart disease: findings from the Scandinavian Simvastatin Survival Study (4S). Atherosclerosis 205, 202-206 (2009).

44. Kendrick, J. et al. Effect of lovastatin on primary prevention of cardiovascular events in mild CKD and kidney function loss: a post hoc analysis of the Air Force/Texas Coronary Atherosclerosis Prevention Study. Am. J. Kidney Dis. 55, 42-49 (2010).

45. Kimura, S. et al. Randomized comparison of pitavastatin and pravastatin treatment on the reduction of urinary albumin in patients with type 2 diabetic nephropathy. Diabetes Obes. Metab. 14, 666-669 (2012).

46. Kinouchi, K., Ichihara, A., Bokuda, K., Morimoto, S. \& Itoh, H. Effects of adding ezetimibe to fluvastatin on kidney function in patients with hypercholesterolemia: a randomized control trial. J Atheroscler. Thromb 20, 245-256 (2013).

47. Koren, M. J. et al. Focused atorvastatin therapy in managed-care patients with coronary heart disease and CKD. Am. J. Kidney Dis. 53, 741-750 (2009).

48. Kouvelos, G. N. et al. The effect of adding ezetimibe to rosuvastatin on renal function in patients undergoing elective vascular surgery. Angiology 66, 128-135 (2015).

49. Lam, K. S., Cheng, I. K., Janus, E. D. \& Pang, R. W. Cholesterol-lowering therapy may retard the progression of diabetic nephropathy. Diabetologia 38, 604-609 (1995).

50. Lee, T. M., Lin, M. S., Tsai, C. H. \& Chang, N. C. Add-on and withdrawal effect of pravastatin on proteinuria in hypertensive patients treated with AT receptor blockers. Kidney Int. 68, 779-787 (2005).

51. Lemos, M. M. et al. Effect of rosuvastatin and sevelamer on the progression of coronary artery calcification in chronic kidney disease: a pilot study. Clin. Nephrol. 80, 1-8 (2013).

52. Mou, S. et al. Pravastatin improves renal progression in patients with chronic glomerulonephritis. Int. J. Clin. Exp. Med. 9, 1732-1739 (2016).

53. Nanayakkara, P. W. et al. Effect of a treatment strategy consisting of pravastatin, vitamin E, and homocysteine lowering on carotid intima-media thickness, endothelial function, and renal function in patients with mild to moderate chronic kidney disease: results from the Anti-Oxidant Therapy in Chronic Renal Insufficiency (ATIC) Study. Arch. Intern. Med. 167, 1262-1270 (2007).

54. Ohsawa, M. et al. Effects of pitavastatin add-on therapy on chronic kidney disease with albuminuria and dyslipidemia. Lipids Health Dis. 14, 161 (2015).

55. Rahman, M. et al. Progression of kidney disease in moderately hypercholesterolemic, hypertensive patients randomized to pravastatin versus usual care: a report from the Antihypertensive and Lipid-Lowering Treatment to Prevent Heart Attack Trial (ALLHAT). Am. J. Kidney Dis. 52, 412-424 (2008).

56. Rutter, M. K. et al. Protection Against Nephropathy in Diabetes with Atorvastatin (PANDA): a randomized double-blind placebocontrolled trial of high- vs. low-dose atorvastatin(1). Diabet. Med. 28, 100-108 (2011).

57. Sawara, Y. et al. Effects of lipid-lowering therapy with rosuvastatin on atherosclerotic burden in patients with chronic kidney disease. Intern. Med. 47, 1505-1510 (2008). 
58. Scanferla, F., Toffoletto, P. P., Roncali, D. \& Bazzato, G. Associated effect of hepatic hydroxymethylglutaryl coenzyme A reductase + angiotensin converting enzyme inhibitors on the progression of renal failure in hypertensive subjects. Am. J. Hypertens. 4, 868 (1991).

59. Shepherd, J. et al. Effect of intensive lipid lowering with atorvastatin on renal function in patients with coronary heart disease: the Treating to New Targets (TNT) study. Clin. J. Am. Soc. Nephrol. 2, 1131-1139 (2007).

60. Takazakura, A. et al. Renoprotective effects of atorvastatin compared with pravastatin on progression of early diabetic nephropathy. Journal of Diabetes Investigation 6, 346-353 (2015).

61. Tonelli, M. et al. Effect of pravastatin on rate of kidney function loss in people with or at risk for coronary disease. Circulation 112, 171-178 (2005)

62. Yasuda, G. et al. Safety and efficacy of fluvastatin in hyperlipidemic patients with chronic renal disease. Ren. Fail. 26, 411-418 (2004).

63. Kimura, G. et al. Effects of atorvastatin on renal function in patients with dyslipidemia and chronic kidney disease: assessment of clinical usefulness in CKD patients with atorvastatin (ASUCA) trial. Clin. Exp. Nephrol. 21, 417-424 (2017).

64. Dalla Nora, E. et al. Atorvastatin improves metabolic control and endothelial function in type 2 diabetic patients: a placebocontrolled study. J. Endocrinol. Invest. 26, 73-78 (2003).

65. Fried, L. F. et al. Lipid modulation in insulin-dependent diabetes mellitus: effect on microvascular outcomes. J. Diabetes Complications 15, 113-119 (2001)

66. Naci, H., Brugts, J. J., Fleurence, R. \& Ades, A. E. Dose-comparative effects of different statins on serum lipid levels: a network metaanalysis of 256,827 individuals in 181 randomized controlled trials. Eur J Prev Cardiol 20, 658-670 (2013).

67. Zoppini, G. et al. Predictors of Estimated GFR Decline in Patients with Type 2 Diabetes and Preserved Kidney Function. Clin. J. Am. Soc. Nephrol. 7, 401 (2012).

68. Hayward, R. A. \& Krumholz, H. M. Three reasons to abandon low-density lipoprotein targets: an open letter to the Adult Treatment Panel IV of the National Institutes of Health. Circ. Cardiovasc. Qual. Outcomes 5, 2-5 (2012).

69. Zhou, Q. \& Liao, J. K. Pleiotropic effects of statins. - Basic research and clinical perspectives. Circ. J. 74, 818-826 (2010).

70. Wanner, C. \& Tonelli, M. KDIGO Clinical Practice Guideline for Lipid Management in CKD: summary of recommendation statements and clinical approach to the patient. Kidney Int. 85, 1303-1309 (2014).

71. Rucker, G., Schwarzer, G., Carpenter, J. R. \& Schumacher, M. Undue reliance on I(2) in assessing heterogeneity may mislead. BMC Med. Res. Methodol. 8, 79 (2008).

72. Lin, J., Knight, E. L., Hogan, M. L. \& Singh, A. K. A comparison of prediction equations for estimating glomerular filtration rate in adults without kidney disease. J. Am. Soc. Nephrol. 14, 2573-2580 (2003).

\section{Acknowledgements}

The authors would like to thank Jan Schoones (Waleaus Library, Leiden University Medical Center) for his indispensable help with the literature search. We also thank Katerina Papadimitropoulou, for advice regarding network meta-analysis methodology.

\section{Author Contributions}

Research idea and study design: K.E., O.D., E.H.; data acquisition: K.E., E.H.; data analysis/interpretation: E.H., O.D., K.E., J.F., F.D.; statistical analysis: K.E., O.D.; supervision and mentorship: J.F., E.H., O.D., F.D. Each author contributed important intellectual content during manuscript drafting or revision and accepts accountability for the overall work by ensuring that questions pertaining to the accuracy or integrity of any portion of the work are appropriately investigated and resolved.

\section{Competing Interests}

The authors declare no competing interests.

\section{Additional information}

Supplementary information is available for this paper at https://doi.org/10.1038/s41598-019-53064-x.

Correspondence and requests for materials should be addressed to K.E.

Reprints and permissions information is available at www.nature.com/reprints.

Publisher's note Springer Nature remains neutral with regard to jurisdictional claims in published maps and institutional affiliations.

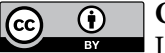

Open Access This article is licensed under a Creative Commons Attribution 4.0 International

License, which permits use, sharing, adaptation, distribution and reproduction in any medium or format, as long as you give appropriate credit to the original author(s) and the source, provide a link to the Creative Commons license, and indicate if changes were made. The images or other third party material in this article are included in the article's Creative Commons license, unless indicated otherwise in a credit line to the material. If material is not included in the article's Creative Commons license and your intended use is not permitted by statutory regulation or exceeds the permitted use, you will need to obtain permission directly from the copyright holder. To view a copy of this license, visit http://creativecommons.org/licenses/by/4.0/.

(C) The Author(s) 2019 